\title{
Control System for Electrical Exploding Wire Opening Switch Experimental Equipment
}

\author{
YANG Jia-zhi ${ }^{1, a}$, DENG Yi-chen, ${ }^{1, b}$ and XIAO Peng ${ }^{1, c}$ \\ 1 Department of Information Science and Engineering, GuiLin University of Technology, GuiLin, \\ GuangXi,China 541004 \\ ajiazhi_yang@126.com, b104238327@qq.com, '390121367@qq.com
}

\begin{abstract}
Keywords: Wire electrical explosion, Opening switches, Experimental equipment, Control system.

Abstract. The control system of experimental equipment for electrical exploding wire opening switches designed in this paper includes two parts, i.e., the main control system and human-machine interface. The main control system is composed of a c8051f020 MCU motherboard and two pieces of IO expansion boards to achieve functions as controlling the boosting-rectifying-charging circuit, outputting trigger signal to spark gap switch, communicating with human-machine interface, and so on. Human-machine interface is composed of a c8051f020 MCU motherboard and a piece of IO expansion board. The function of the human-machine interface is to response the operator's instructions, display experimental parameters, communicate with main control system. The test results of the control system of experimental equipment for electrical exploding wire opening switches show that the control system can control discharging voltage precisely, perform opening switch experiments and acquire experimental data. Meanwhile, the control system has the advantage of high degree of automation and system security.
\end{abstract}

\section{Introduction}

The experimental device for electric exploding wire opening switch involves three aspects about high pressure, strong current and complex electromagnetic environment.In terms of energy storage density, inductive energy storage is more practical for pulsed power systems which is demanding of volume and efficiency ${ }^{[1-4]}$. Its function needs to be to meet the requirements of metal wire electrical explosion experiment. Transient voltage reached tens of KV in the explosion experiment, So in the design of the overall experimental equipment to take into account the security issues. So we need a control circuit to measure and control the electrical parameters in the discharge process of the pulsed power system.

In view of the above situation, a control system of experimental device for electric exploding wire opening switch is designed in this paper. The experiment device comprises a man-machine interface and a main control circuit.

\section{The overall design of the control system}

Wire electrical explosion break switch experiment device control system schematic diagram is shown in figure 1. 


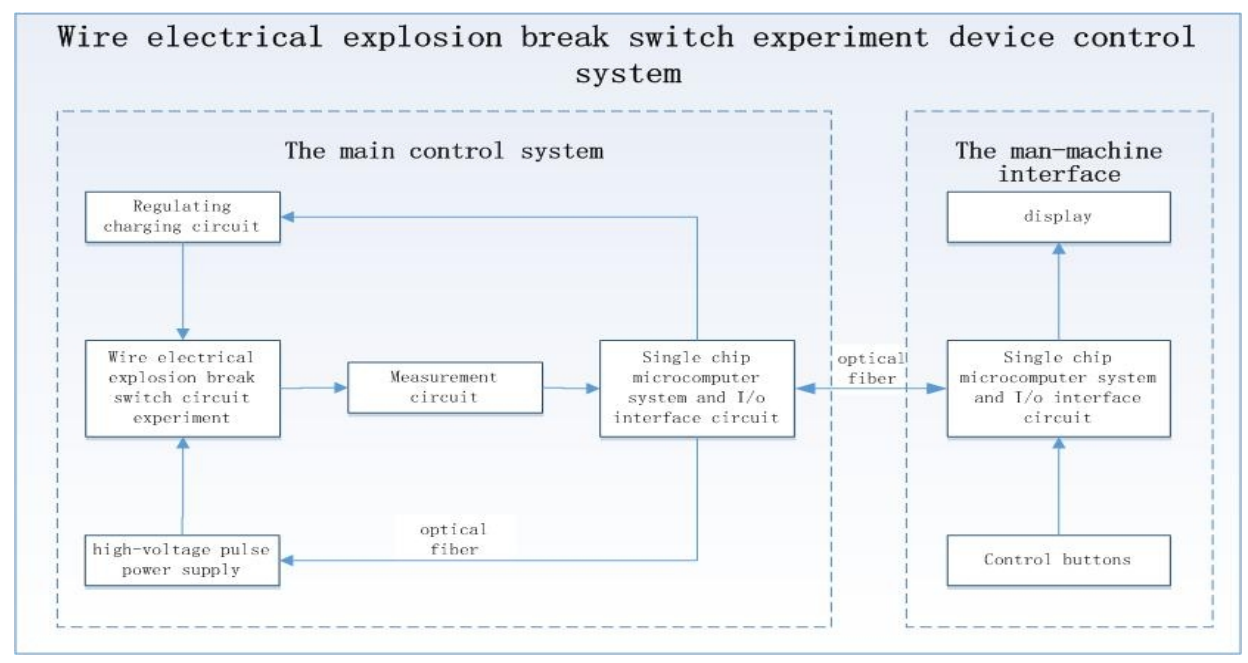

Fig.1 Wire electrical explosion break switch experiment device control system

The control system of experimental device for electric exploding wire opening switch is mainly composed of the main control system and human-machine interface. Human machine interface is responsible for the display data and operation function. The existence of the man-machine interface is for the convenience of the operation personnel of laboratory equipment for safe operation and make the high-pressure and low-pressure separation. First, make sure the safety of the experiment,the next, through the man-machine interface Settings need to reach the specified values of charging pulse capacitor group, and then to charge, discharge and so on a series of actions. On the main control system, its working process can be summarized as follows, first of all to be able to response from the man-machine interface of the operation instructions, collecting voltage value, receives the default pulse charging capacitance values and give the current parameters to the man-machine interface display. Secondly it to control the high voltage relay, sending trigger circuit of the trigger signal, the control of dc motor drive to control the electric voltage regulator, read through high voltage measuring circuit to measure the current pulse capacitor voltage and through the output signal to control the electric voltage regulator surge tank and then by using closed-loop control until the pulse capacitor charging to the default values.Again, when the charging is completed,the main control system with human-machine interface by optical signal transceiver and through the way of produce low voltage pulse signals in response to a discharge instructions from the man-machine interface ${ }^{[5]}$, Finally, by using high voltage pulse power to zoom in low voltage pulse power form a peak for tens of thousands of volts high-voltage pulse signal and spark gap switch of trigger electrical explosion wire break switch circuit experiment is on state, so as to complete a circuit breaker experiment of electric exploding wire.

\section{The implementation scheme of the control system}

a. The hardware design of the system

The control system is mainly composed of the main control system and human-machine interface.

In terms of the main control system,the main circuit of electric explosion of electric exploding wire opening switch experiment circuit is composed of electrical explosion wire clamp, its load circuit is composed of water resistance and air switch.Explosion wire break switch experiment circuit principle diagram is shown in figure 2 . 


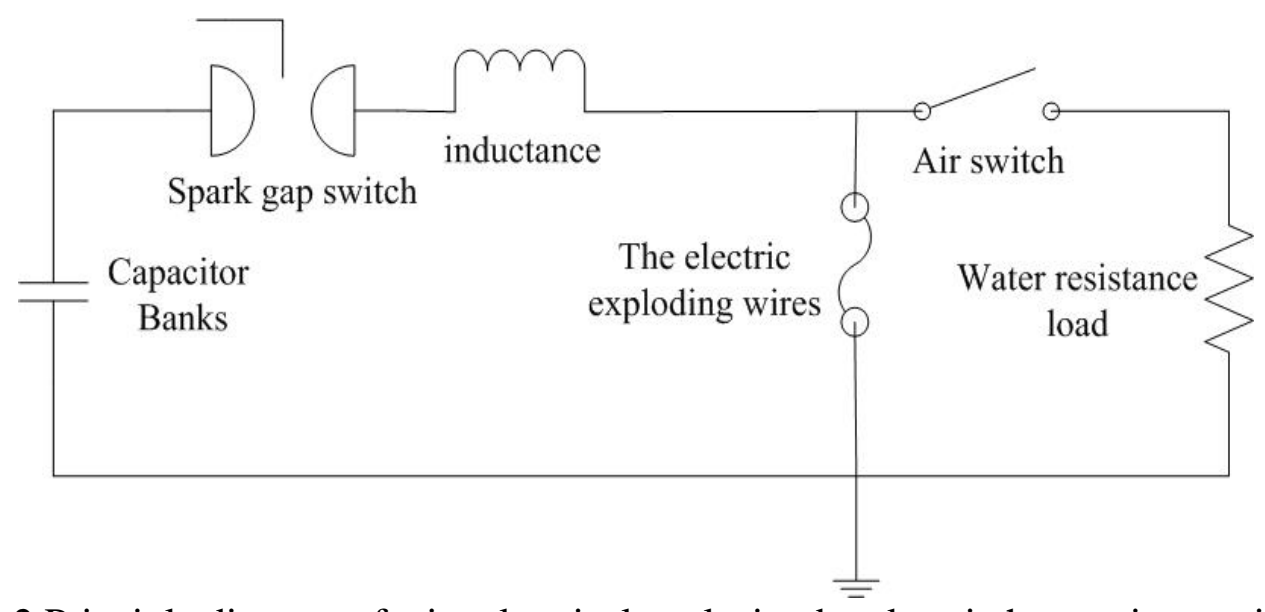

Fig.2 Principle diagram of wire electrical explosion break switch experiment circuit

In the trigger circuit, spark gap switch is the key part. As is shown in,A high voltage trigger signal is issued after charging the capacitor group to the experimental preset value,then the spark gap switch conduction and making the circuit including capacitor group, spark gap switch, inductance, electric exploding wire form a loop so as to ensure the power of capacitor group can through the loop is loaded into the electric exploding wires, as the same time,temperature and resistance of exploding wires continuously increase sharply until exploding wire immediately cut off. At this point the original circuit to break and the air gap switch of the load circuit be breakdown form connected circuit.In this case, all the energy stored in the loop inductance is in the form of a pulse load in the load $^{[6]}$.

The main control system use c8051f020 as the control board and Its role is to collect the voltage of capacitor banks, control trigger circuit and data transfer with man-machine interface.

The main control system principle diagram is shown in figure 3 .

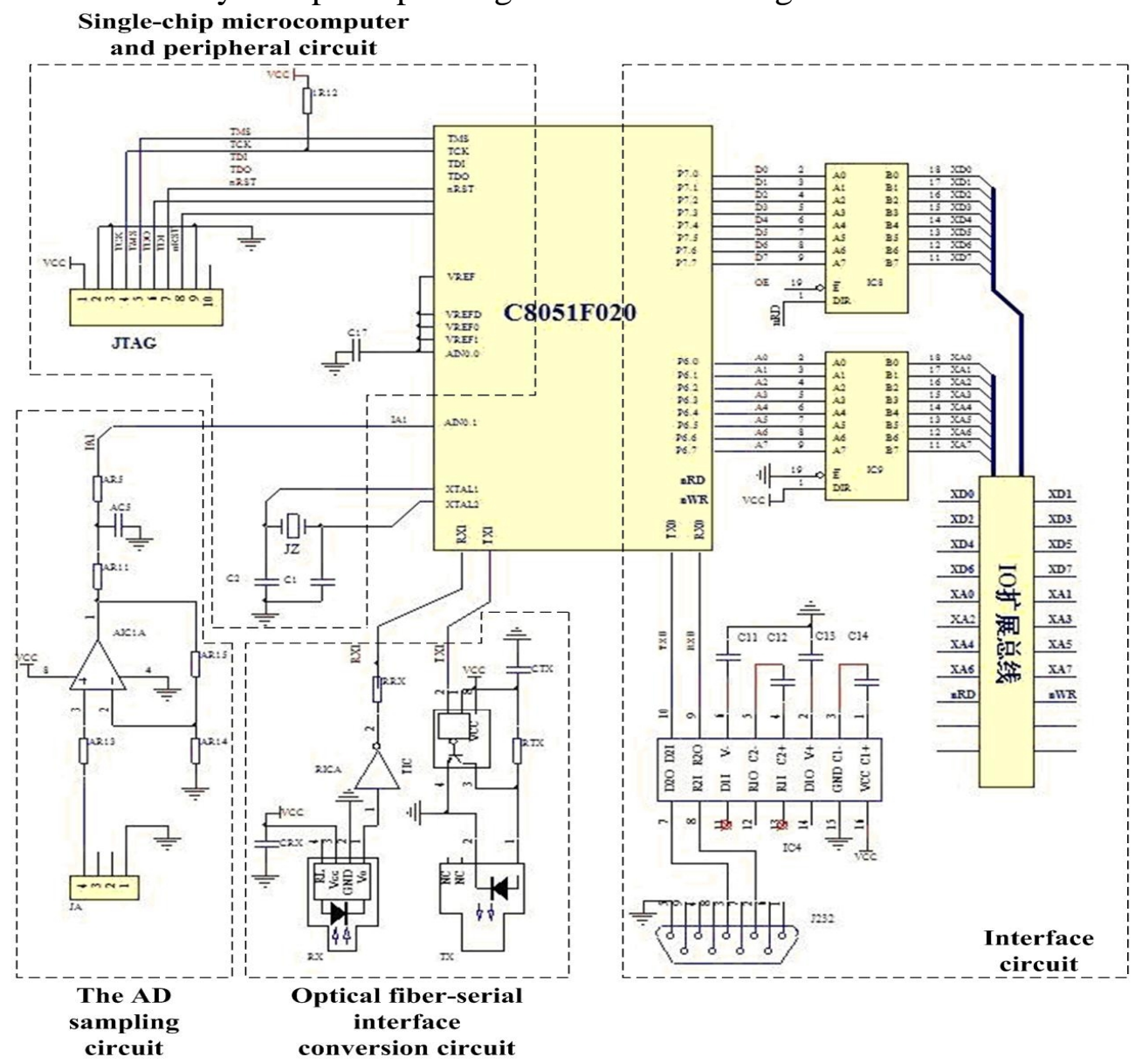

Fig.3 Principle diagram of the main control circuit 
In terms of the man-machine interface, Its main uses optical fiber serial port and the main control for data transmission ${ }^{[7]}$. The function of the man-machine interface is to display data and operation and control DC motor driver through operation button of human machine interface so as to drive the electric voltage regulator to change the voltage to the required value of the experiment.In the aspect of chip control,man-machine interface and the main control system are made based on c8051f020 as the control panel. The man-machine interface as the main auxiliary control of the control system,Its role is mainly to send the operation information to the master control terminal and accept the main control data and display on the display screen of dot matrix LED.

b. The software design of the system

The system software flow chart is as follows:

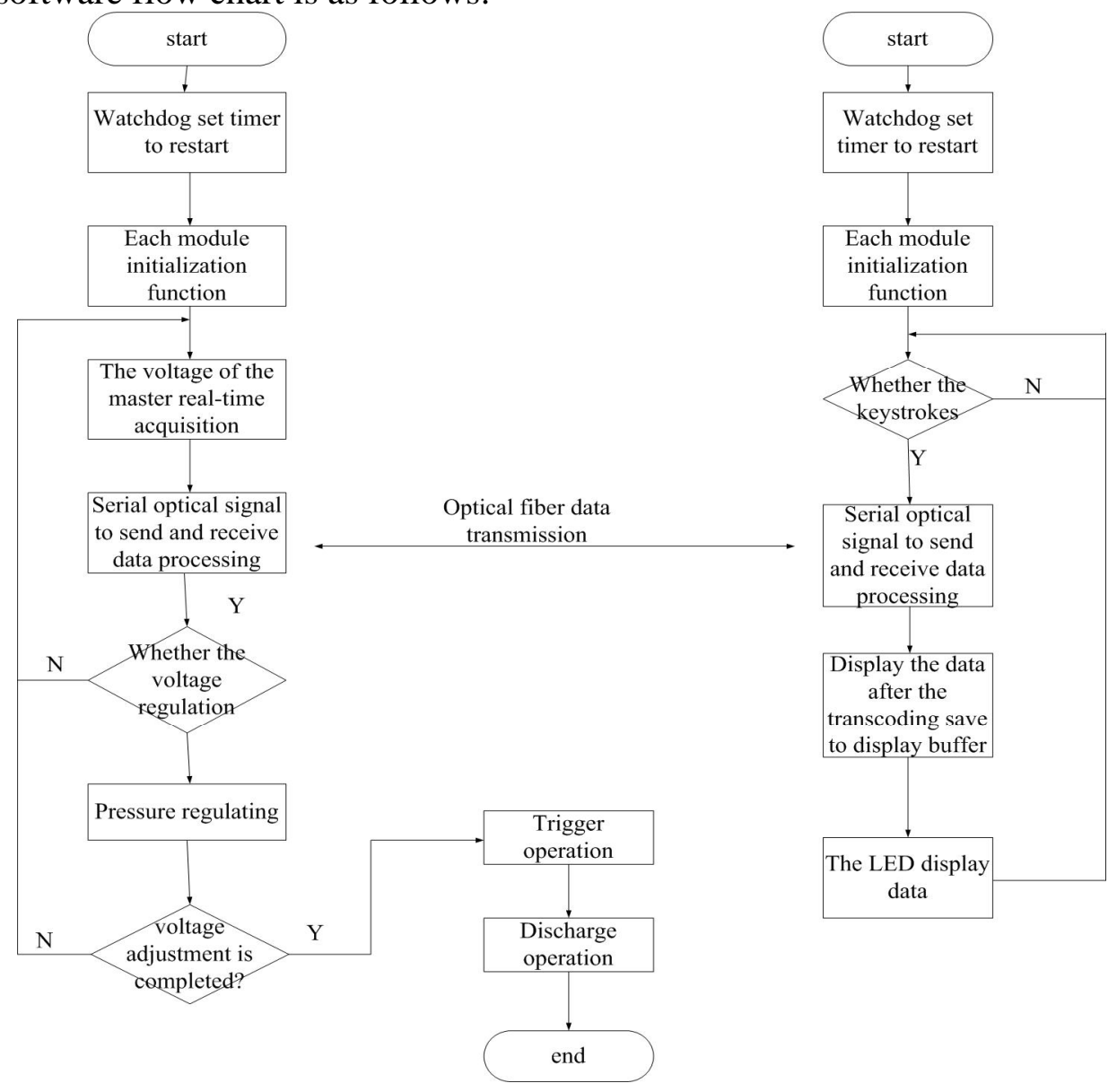

Fig4 The software flow chart main control system (left) and man-machine interface (right)

Figure 4 is the software flow chart about main control system and human-machine interface which set the watchdog and module initialization for master control terminal and human-computer interaction are indispensable steps. The purpose of the watchdog set up time to restart is help restart and avoid problems in the process of regulating when the MCU or program is not running. Initialization module function includes two parts, one part is the internal cross port configuration of C8051F020 and the set of basic functions such as reference voltage source and clock and so on. The other part is initialization for external hardware initial state controlled by MCU, including the voltage regulator to the minimum, LED display data reset, high voltage relay set the initial state, etc. These Settings are mainly guarantee the initial line is in a state of a security.

\section{The safety of the control system design}

Because the wire electrical explosion experiment device relates to high voltage, strong current and devices with complex electromagnetic environment, it will have strong electromagnetic interference on low voltage control circuit.So when designing experiment device control system should 
guarantee the safe operation of the whole system.Measures include a good grounding system, shielding of low voltage circuit, isolate the high and low voltage and optical fiber communication between the main control system and the human computer interface.

i) Ground: The entire electrical explosion experiment device placed in a metal cabinet and surround the whole experiment device with metal net and the metal mesh is connected to the earth to play a role in isolation.

ii) Shield: The main control circuit board is shielded by the aluminum shell and the aluminum shell connected to the earth.Similar measures are also used in other easily subject to electromagnetic interference to reduce the interference.

iii) Isolation: Circuit control signals and trigger signals using photoelectric coupling isolation and the power supply circuit of trigger circuit and human-computer interaction end use isolated transformer for power supply.

iv) Optical fiber communications:Man-machine interface and the communication between the main control system is done using the serial port UART1 of the chip 8051 f020 through a light signal transceiver and fiber.

\section{Conclusions}

In this paper, a control system of the experiment device of electric exploding wire opening switch is designed. The system is mainly composed of the main control system and man-machine interface. At the same time, the reasonable design of the control system software and hardware, take the grounding, shielding, isolation, optical fiber communication and other measures. Because the experiment device experiment device reliable operation, high automation and data is accurate, the experimental device can provide experiment platform for the application of the electric explosive circuit breakers.

\section{Acknowledgements}

This work was financially supported by the Natural Science Foundation of China (51167004), GuangXi Natural Science Foundation (2013GXNSFBA019250, 2011GXNSFA08022).

\section{References}

[1] Senthil K, Mitra S, Roy A, et al. Compact inductive energy storage pulse power system[J]. Review of Scientific Instruments, 2012, 83(5):054703.

[2] Takaki K, Yagi I, Fujiwara T, et al. Influence of circuit parameter on ozone synthesis using inductive energy storage system pulsed power generator[J]. IEEE Transactions on Dielectrics and Electrical Insulation, 2011, 18(5): 1752-1758.

[3] Shi-rong hao, Wei-ping Xie, bo-nan ding, et al. Marx generator driven inductive energy storage type pulse power source [J]. High voltage technology, 2009, 35 (3) : 657-660(in Chinese).

[4] Wei-ping Xie, shi-rong hao, min-hua wang, et al. A compact high power pulse source based on inductive energy storage technology [J]. High voltage technology, 2004, 30 (1) : 41 and 42(in Chinese).

[5] Stephens J, Mischke W, Neuber A. The impact of wire environment on electro-explosive fuse performance[J]. IEEE Transaction on Plasma Science, 2012, 40(10): 2517-2522.

[6] Jia-zhi Yang. Wire electrical explosion of circuit breaker tripping time experimental study [J]. High voltage technology, 2015, 41 (6) : 1-6(in Chinese).

[7] Zheng-Ying Li. Pulse power technology: 1 edition [M]. Beijing: water conservancy electric power press, 1992(in Chinese) 